J Phys Chem Lett. 2016 July 07; 7(13): 2496-2501. doi:10.1021/acs.jpclett.6b00940.

\title{
Dark State-Modulated Fluorescence Correlation Spectroscopy For Quantitative Signal Recovery
}

\author{
Jung-Cheng Hsiang§ ${ }^{\S}$ Blake Fleischer§, and Robert M. Dickson* \\ School of Chemistry \& Biochemistry and Petit Institute for Bioengineering and Bioscience, \\ Georgia Institute of Technology, Atlanta, GA 30332-0400
}

\begin{abstract}
Excitation of few-atom Ag cluster fluorescence produces significant steady-state dark state populations that can be dynamically optically depopulated with long wavelength co-illumination. Modulating this secondary illumination dynamically repopulates the ground state, thereby directly modulating nanodot fluorescence without modulating background. Both fast and slow modulation enable unmodulated background to be quantitatively removed in Fluorescence Correlation Spectroscopy (FCS) through simple correlation-based averaging. Such modulated dual-laser FCS enables recovery of pure Ag nanodot fluorescence correlations even in the presence of strong, spectrally overlapping background emission. Fluorescence recovery is linear with Fourier amplitude of the modulated fluorescence, providing a complementary approach to backgroundfree quantitation of modulatable emitter concentration in high background environments. Using the expanding range of modulatable fluorophores, such methodologies should facilitate biologically relevant studies in both complex autofluorescent environments and multiplexed assays.
\end{abstract}

\section{Graphical Abstract}

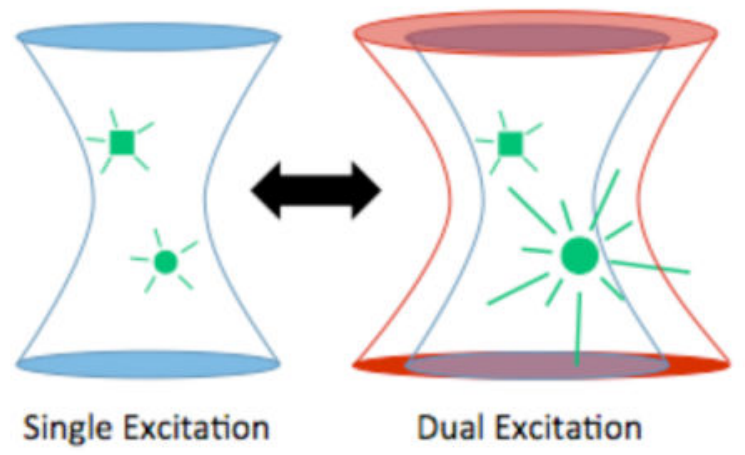

Fluorescence labeling and detection enable high sensitivity and fast temporal resolution, often revealing important structures and dynamics in materials and biological imaging.

\footnotetext{
$\S_{*}$ These authors contributed equally to this work *dickson@chemistry.gatech.edu

Supporting information. Expanded plots of traces in Figure 3 and modulation depth vs. modulation frequency response curves for the $630 \mathrm{~nm}$ emitting Ag nanocluster with scattered light controls are provided.
} 
Autofluorescence from endogenous fluorophores, however, too often obscures fluorescence of interest and can bias data analyses. To improve sensitivity in biological imaging, many groups have focused on making brighter fluorophores. ${ }^{1-3}$ Unfortunately, the brightness of molecular-scale emitters is limited by the number of electrons involved in the optical transition. As organic dyes routinely approach this oscillator strength limit, ${ }^{4}$ the greatest sensitivity gains in high-background biological imaging are more likely to result from background reduction. Although passive shifting of excitation and emission to longer wavelengths is the most straightforward way to avoid autofluorescence, ${ }^{5-7}$ this limits the spectral range and number of emitters that can be used. Analogously, long-lived ( $\mu \mathrm{s}-\mathrm{ms})$ emitters can be utilized with pulsed excitation and time-gated detection to reject fastdecaying emission from both more standard and endogenous fluorophores. ${ }^{8}$ Reducing background in this manner enables resolution of different species based on their luminescent lifetimes, but such long-lived luminophores typically suffer from low excitation and emission rates, resulting from weak connections between ground and emissive states.

Originally demonstrated with DNA-encapsulated Ag clusters ("Ag nanodots"), ${ }^{9-10}$ synchronously amplified fluorescence intensity recovery ("SAFIRe") utilizes the photoinduced build-up of dark state population that can be optically depopulated with longwavelength co-illumination (Fig. 1). Because this secondary long-wavelength coillumination rapidly repopulates the ground state, dual-laser excitation of Ag nanodots yields brighter steady-state emission than that achieved with primary laser excitation alone. ${ }^{9}$

Although Ag nanodots naturally produce strong, photostable emission compared to conventional organic dyes, it is their moderate dark state quantum yield $(\sim 1 \%)$ and dark state lifetime ( $>10 \mu \mathrm{s})$ that enable the dark state to achieve significant populations under typical confocal excitation intensities. ${ }^{11-13}$ Direct, long-wavelength excitation of this dark state increases fluorescence by shifting steady-state population back to the emissive manifold more rapidly than achieved through natural dark-state decay. ${ }^{9,}{ }^{14}$ Such reversible modulation of ground/dark state populations under single and dual laser excitation directly modulates the total fluorescence at the secondary laser modulation frequency. Fourier transformation of the collected fluorescence reveals a peak at the secondary modulation frequency that is shifted away from all (unmodulated) background emission, enabling demodulation to selectively recover only the desired fluorophore signal. ${ }^{7,9,14}$ Enhancements of sensitivity then result from background removal, showing up to 100-fold improvements in signal recovery. 9,14

SAFIRe works on both single molecule and bulk samples to linearly recover relative concentrations of modulatable fluorophore signals from high background. ${ }^{10}$ In contrast, fluorescence correlation spectroscopy (FCS) typically requires very low concentrations $(\sim \mathrm{nM})$ to recover fluctuation-based diffusion, concentration, and dark state dynamics information, in conjunction with very low background environments. ${ }^{15}$ It is this background emission that typically precludes performing FCS in many biologically relevant systems. ${ }^{6}$ Although the correlation fitting model can be modified to include background emission, ${ }^{16}$ account for laser fluctuations, ${ }^{17}$ or extract photophysics, ${ }^{18}$ low analyte of interest concentrations and relatively low background conditions must be maintained. ${ }^{19}$ Even with weak background present, the true number of molecules inside the focal volume is not directly recoverable as intensity fluctuations arise from both signal and background emitters. 
Herein, we utilize the optical modulation of Ag nanodots to develop new bulk spectroscopic correlation methods that improve on standard one-laser FCS methods to quantitatively recover fluorophore concentration and dynamics even in high background environments.

\section{Materials and Methods}

Analogous to our previous reports, ${ }^{11-12,20}$ we prepared a new $630 \mathrm{~nm}$-emitting Ag nanodot that exhibits a higher dark state quantum yield than other nanodot species. This new emitter is readily synthesized using our published procedures in aqueous solution, ${ }^{11}$ but using the ss-DNA sequence 5'-CCCCAACTCC-3' as the template. Specifically, ss-DNA (Integrated DNA Technologies) is first diluted in water to form a final $1 \mathrm{~mL}, 20 \mu \mathrm{M}$ solution. $\mathrm{AgNO}_{3}$ (Sigma-Aldrich, 204390) is added in a 11:2 Ag:DNA molar ratio via a $2 \mathrm{mg} / \mathrm{mL}$ aqueous solution. Next a 6:11 $\mathrm{BH}_{4}: \mathrm{Ag}$ molar ratio of $1 \mathrm{mg} / \mathrm{mL} \mathrm{NaBH}_{4}$ (Sigma-Aldrich, 213462) is added to the ss-DNA/Ag mixture within 30 seconds of combining $\mathrm{NaBH}_{4}$ and water. The resulting $1 \mathrm{~mL}$ mixture is then vortexed for $\sim 1$ minute, incubated at room temperature for ten hours and then refrigerated $\left(\sim 4^{\circ} \mathrm{C}\right)$ for permanent storage. While Ag clusters may continue to form for approximately 24-48 hours, they are stable in stock concentrations for at least two weeks.

Aqueous Ag nanodot samples are illuminated by spatially overlapping primary excitation $(594 \mathrm{~nm})$ and secondary $(805 \mathrm{~nm})$ continuous wave $(\mathrm{cw})$ lasers at the focal plane of an optical microscope (Olympus IX71, 60x 1.2NA water immersion objective). Both primary and secondary wavelengths are filtered out and $630 \mathrm{~nm}$-centered fluorescence is collected through a bandpass filter (Semrock). The secondary laser is intensity-modulated with a square wave pattern by an electro-optical modulator (Conoptics, 360-80). Fluorescence is collected through a $100-\mu \mathrm{m}$ multimode fiber and routed to an avalanche photodiode (PerkinElmer, SPCM-AQRH-15-FC). Time-stamped photon arrival times are recorded with a National Instruments PCI-6602 fast counting card along with a BNC 2121 connector block, and photon-by-photon correlations are performed with custom scripts/in-house software written in C++, compiled, and called from MATLAB 2015a (Fig. S1).

\section{Properties of Ag clusters}

Common to all $\mathrm{Ag}$ nanodot emitters, primary-only excitation yields bright fluorescence,

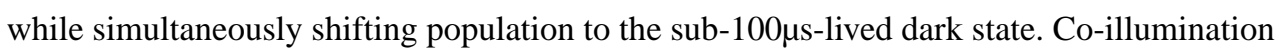
with wavelengths from 680 to $950 \mathrm{~nm}$ repopulates the ground state faster than the dark state naturally decays, thereby increasing steady-state emission. ${ }^{9}$ Thus, all nanodots exhibit varying degrees of enhanced fluorescence under secondary co-illumination, but with varying degrees resulting from differences in steady-state dark state populations. ${ }^{12}$ We report a new, spectrally pure Ag nanodot emitter synthesized with 5'-CCCCAACTCC-3' single stranded DNA with 560nm/630nm absorption/emission maxima, shown in Fig. 1. The emission spectrum is independent of excitation wavelength (Fig S2), further confirming that a single species is obtained. As measured by FCS and bulk fluorescence, this $630 \mathrm{~nm}$ emitter exhibits a dark state quantum yield of $5 \%$ and a fluorescence quantum yield of $18 \%$ (Table 1 ). The photophysical on and off times (residence time in bright and dark manifolds, respectively) determine the maximum modulation frequency attainable, as steady-state populations must 
be able to be established during a single modulation cycle. ${ }^{14,21}$ The high dark state formation rate, $\mathrm{k}_{\mathrm{d}}$, enables $\mathrm{Ag}$ nanodot steady-state populations to be established very quickly, allowing modulation periods both much faster and much slower than the diffusion time to be utilized. Thus, both slow $(1 \mathrm{~Hz})$ and fast $(100 \mathrm{kHz})$ modulation schemes can be employed using SAFIRe to develop dual-laser modulated fluorescence correlation (FCS) measurements that recover analyte signals from high background samples. While both schemes are able to subtract background signals, the low frequency modulation is important for samples with SAFIRe response slower than diffusion, as the necessary build-up of dark state population would occur too slowly for fluorescence enhancement to be observed with fast modulation. In all modulatable fluorophores, the Fourier transform provides a means for concentration recovery, provided calibrations are performed.

Correlation functions are calculated from photon arrival trajectories to naturally average over many individual events and report on characteristic timescales of the system under study. ${ }^{18}$ As with any FCS experiment, fluctuations resulting from individual molecules diffusing into the focal volume must be discernable, mandating relatively low concentrations, bright analyte molecules, stable lasers, sensitive detectors, and very low obscuring background. ${ }^{16-17,22}$ In the limit of zero background, the number of molecules in the excitation volume is determined using an autocorrelation fit to a standard diffusing molecule model:

$$
G_{2}(\tau)=1+\frac{1}{N} \cdot \frac{1}{\left(1+\frac{4 D \tau}{\mathrm{w}_{x y}{ }^{2}}\right)} \cdot \frac{1}{\sqrt{1+\frac{4 D \tau}{\mathrm{w}_{z}{ }^{2}}}}
$$

in which $\tau$ is the delay, $\mathrm{N}$ is the average number of molecules in the focal volume, $\mathrm{D}$ is the diffusion coefficient and $\mathrm{w}_{\mathrm{xy}}, \mathrm{w}_{\mathrm{z}}$ are the excitation volume dimensions. ${ }^{19,}{ }^{23}$ Underlying dynamics that lead to fluorescence fluctuations are not synchronized, leading to increased contrast in the autocorrelation for decreasing numbers of molecules contributing to the overall signal. However, signal to noise of the correlation curve increases with analyte concentration. ${ }^{16-17,19}$ Low background is crucial to recovering signals of interest at any analyte concentration. Even recent high concentration FCS investigations that subtract out excitation laser fluctuations ${ }^{17}$ would still suffer from unwanted background obscuring the number of emitters from being accurately determined.

\section{SAFIRe-FCS}

Fluorescence from mixed Ag nanodot and Texas red solutions was recorded under $\mathrm{cw} 594$ $\mathrm{nm}$ and intensity-modulated $805 \mathrm{~nm}$ co-excitation (modulation frequencies of $100 \mathrm{kHz}$ or 1Hz, Fig. 3). The externally applied square-wave modulation period defines the microtime histogram window, with the secondary laser illuminating the sample for only half of each period. The microtime histogram exhibits two regimes - higher fluorescence intensity with secondary laser on, and lower fluorescence intensity with secondary laser off (Figs. 3A and $3 \mathrm{~B})$. As the secondary laser is much longer wavelength $(805 \mathrm{~nm})$ than is the collected emission $(630 \mathrm{~nm})$, the non-modulatable background is unchanged (Poisson distributed) throughout the entire microtime window. At modulation frequencies that are high relative to 
the diffusion rate through the focal volume, the same signal and background emitters are probed with both one- and two-laser halves of each period, as molecules have had insufficient time to diffuse out of the focal volume during the modulation period. Representing the signal from primary only excitation as $\mathrm{S}(\mathrm{t})$ and the obscuring background $\mathrm{B}(\mathrm{t})$, there is a relative enhancement factor, $\mathrm{c}$, of the signal as a result of secondary coillumination $(\mathrm{cS}(\mathrm{t}))$. The enhancement does not affect the non-modulated background emission, which is always present. This leads to two regimes of signal: $\mathrm{S}(\mathrm{t})+\mathrm{B}(\mathrm{t})$ and $\mathrm{cS}(\mathrm{t})$ $+\mathrm{B}(\mathrm{t})$, resulting from single- and dual-laser excitation, respectively. The relative enhancement, $\mathrm{c}$, is greater than or equal to 1 , where a value of $\mathrm{c}$ equal to 1 corresponds to no enhancement over primary excitation alone. Because, on average, the background is the same in the two halves of the modulation period, one can use the fact that correlations average over large amounts of data to mathematically extract the signal of interest in FCS. By splitting the intensity trajectory to yield two parallel trajectories consisting of 1) the photons collected with primary-only excitation and 2) those collected with dual excitation, the traces can be processed to extract the pure signal autocorrelation curve without background contributions, according to Equation 2:

$$
\begin{gathered}
\int_{-\infty}^{\infty}(c S[t]+B[t])(c S[t+\tau]+B[t+\tau]) t+\int_{-\infty}^{\infty}(S[t]+B[t])(S[t+\tau]+B[t+\tau]) t \\
-\int_{-\infty}^{\infty}(c S[t]+B[t])(S[t+\tau]+B[t+\tau]) t-\int_{-\infty}^{\infty}(S[t]+B[t])(c S[t+\tau]+B[t+\tau]) t \\
=(c-1)^{2} \int_{-\infty}^{\infty} S[t] S[t+\tau] t
\end{gathered}
$$

In Equation 2, the trace is separated into longer traces consisting of the photons arriving in each half period, then the two cross correlations are subtracted from the sum of the autocorrelations of each half-trace. All correlations must remain unnormalized until the final result is obtained, yielding the pure signal, $\mathrm{S}(\mathrm{t})$ autocorrelation without background.

With sufficient averaging, the correlation subtraction of Eq. 2 recovers the true autocorrelation curve of the background-free signal of interest. Because photons are separated from the original time trace, Nyquist sampling dictates the fastest time that can be probed in the correlation-subtracted curve to be twice the modulation period. Because the dark state is modulated, modulation depth decreases at higher frequencies, forcing one to modulate more slowly than the $(\sim 11 \mu \mathrm{s})$ dark state lifetime. As the modulation period is still much faster than is diffusion through the focal volume, fitting the recovered signal correlation to Eq. 1 allows for extraction of the number of molecules and diffusion time, without being obscured by either Ag nanodot dark state dynamics or Texas Red emission (Fig. 3). At both high and low modulation frequencies, correlation subtraction according to Eq. 2 (Figs. 3C and 3D) reveals higher correlation amplitudes than present in the raw FCS curves. Correlated background emission common to both halves of the modulation period is contained within the cross correlations, and these are subtracted from the signal + background autocorrelations in each half period. This correlated background subtraction is what results in the higher correlation amplitude when the true signal correlation is recovered in SAFIRe-FCS (Equation 2).

In principle, correlation subtraction works best at very high modulation frequencies. In this regime, the molecules generating background and those giving signal and enhancement have 
not diffused out of the laser focus even after several modulation cycles. Thus, molecule number fluctuations can be quantitatively averaged and subtracted out of the resulting correlations. In fact, the cross correlation amplitudes between the dual and single laserexcited fluorescence portions of each modulation cycle are much higher at high frequency because the exact same emitters are present in both halves of each high frequency modulation cycle. This leads to a more exact background subtraction than possible with low modulation frequencies, which exhibit essentially zero cross correlation amplitude (Fig. $3 \mathrm{CD}$, Fig. S3). In practice, however, such high modulation frequencies require very high emission rates for good signal to noise. For high-frequency modulation, the photon count rate is divided by two as traces are separated into single-laser and dual-laser-excited intensity trajectories. Because signal to noise in correlation functions at a given delay scales with the square of the count rate, this count rate reduction through trace separation, coupled with the multiple operations on the auto and cross-correlations that need to be performed (Eq. 2), further increase noise in the recovered "background-free" correlation. While a unique aspect of these Ag nanodot emitters is their ability to be optically modulated at high frequencies while maintaining large modulation depths, only a limited number of fluorophores exhibit the ability to very rapidly establish optically sensitive steady-state populations that may be depopulated via long wavelength co-excitation. ${ }^{9}, 24$

Low frequency modulation both complements and offers advantages over high frequency modulation-based signal extraction (Fig. 3). Unlike high frequency modulation, low frequency modulation does not reduce sensitivity by dividing the photon count rate. Additionally, no additional Nyquist sampling constraints are imposed when separating dual and single-laser excitation traces. As a result, faster dark state timescales are readily preserved in low frequency correlation subtractions (Fig. 3). Only the long time correlations are sacrificed, but these are much longer than the diffusion time, and thus no diffusive information is lost. Modulating at $1 \mathrm{~Hz}$, photon arrival trajectories from each half period are correlated and combined according to Eq. 2 to recover the background-subtracted correlation, $\langle\mathrm{S}(\mathrm{t}) \mathrm{S}(\mathrm{t}+\mathrm{t})\rangle_{\mathrm{t}}$, where $\langle\ldots\rangle_{\mathrm{t}}$ denotes integration over time. These subtracted correlations can then be averaged over the many modulation cycles, further reducing noise. As with the high frequency modulation, diffusion times are readily recovered, but only average background is subtracted, as single- and dual-laser cross correlations have nearly zero amplitude above background. Thus, molecule number fluctuations are more effectively recovered with high-frequency modulation, if the analyte can support it. By using sufficiently long periods to average over background concentration fluctuations, improved averaging and subtractions are likely to be obtained. Unfortunately, only average, uncorrelated background is removed using low-frequency modulation (Fig. 3D). While low frequency modulation removes background, the cross correlations between enhanced and non-enhanced signals are low, suggesting that additional calibration may be needed to determine the correct analyte molecule concentration in solution.

Although Eq. 2 is general for recovering modulatable fluorophore correlation curves with reduced background, the practical challenges in subtracting background at the different timescales suggest a simpler approach. As the auto and cross correlations are related to Fourier transforms by the convolution theorem, one can extract the number of analyte molecules from the Fourier transform amplitude at the modulation frequency. We have 
shown that this amplitude scales linearly with the total number of modulatable molecules and is shifted to its own unique detection frequency, free from obscuring background signals. ${ }^{9-10,14,21,25}$ While this amplitude is linear in the number of molecules, the slope of Fourier amplitude vs. concentration of modulatable species is a product of many photophysical and experimental parameters. Thus, accurate numbers of molecules can only be obtained from the Fourier amplitude if calibrated by the correlation subtracted SAFIReFCS or through a predetermined calibration curve obtained under identical conditions.

To demonstrate concentration recovery of Ag cluster signals over an unmodulated background, solutions exhibiting constant Texas Red background and varying Ag cluster concentration were prepared. Nanodot signal recovery was performed in solutions ranging from 0.1:1 to 2:1 of Ag cluster to Texas red fluorescence ratios (Fig. 4A), with $100 \mathrm{kHz}$ and $1 \mathrm{~Hz}$ modulation frequencies. Concentration recoveries were performed in the same manner as in Fig. 3. By modulating at $1 \mathrm{~Hz}$, photons are collected for 0.5 seconds with dual laser excitation and 0.5 seconds with primary only excitation. Enough photons are collected to enable calculation of entire correlation functions within each half-second period. Clearly, the $1-\mathrm{Hz}$ recoveries drastically improve correlation contrast through correlation subtraction, since low modulation frequency count rates are not divided as they are at high modulation frequencies. The amplitudes of the cross correlations between modulated and unmodulated halves of the modulation period are much lower, as the background in each half-period is not exactly identical as it is at high frequencies. Thus, $1 \mathrm{~Hz}$ data may not quantitatively recover concentrations, but it does demonstrate the utility of correlation subtractions to remove background signals.

Because SAFIRe induces high fluorescence enhancement, fluorescence is modulated at the secondary laser modulation frequency. Thus, for any specific modulation frequency and excitation conditions, a calibration of Fourier amplitude to known concentration (including extrapolation of Fourier amplitude to zero concentration) provides a convenient methodology for relating modulation-dependent fluorescence signals to true concentration in complex environments. A simple calibration curve thereby enables concentrations to be determined from the Fourier amplitude at both high and low modulation frequencies. The slope of the Fourier transform vs. concentration is related to the frequency-dependent modulation depth (Fig. S4), as modulation frequencies approaching the lifetime of the dark state decay result in decreasing modulation amplitudes. Because modulation shifts $\mathrm{Ag}$ nanodot signals to a unique detection frequency, background subtraction (e.g. Eq. 2) is unnecessary, as background is avoided in the frequency domain. Thus, modulated FCS enables direct Ag nanodot signal recovery through either correlation subtraction (Fig. 4A) or Fourier amplitude calibration (Fig. 4B), promising extraction of analyte concentration for fluorophores with optically modulatable dark states at a wide range of modulation timescales. The correlation subtraction improves sensitivity in all cases, but at low frequencies, only average background is subtracted. At high frequencies, the exact same background is subtracted from each modulation period, but greater measurement noise is obtained. This is why the cross correlation curves are so different in the two cases. Thus, while correlation contrast improves in both cases, the linearity of the Fourier transform is preferred for quantifying concentrations at both low and high frequencies. 
Ag nanodots provide excellent brightness in both linear ${ }^{26}$ and multiphoton fluorescence, ${ }^{27}$ but it is their dark states that enable selective recovery of their emission without recovering background. ${ }^{7,}{ }^{9-10}$ Using new SAFIRe-FCS on both fast and slow timescales, the highly populated dark states can be optically depopulated with long wavelength co-illumination to repopulate the fluorescent manifold of states. Modulating this secondary laser modulates recovered fluorescence, shifting Ag nanodot emission signals to a unique detection frequency. Modulation frequency is limited by the time required to build up steady-state populations, ${ }^{14,21}$ which is largely governed by the Ag nanodot dark state lifetime. SAFIReFCS with modulation much faster than the diffusional transit time in an FCS geometry provides a means for concentration recovery through correlation-based subtraction of enhanced and unenhanced portions of the modulation cycle. Lower frequency modulation provides incomplete background suppression, as subtractions are on timescales significantly longer than diffusion, and are thus not performed on the same analyte and background emitters. For fluorophores with SAFIRe responses slower than the diffusional transit time through the detection volume, high frequency modulation does not result in fluorescence enhancement because the photophysical dark state populations remain low - diffusion is faster than the time needed to populate them. In this case, only the low frequency scheme provides a means for concentration recovery via correlation subtractions, provided calibrations are made. In both low and high modulation frequencies, the Fourier amplitude provides a linear relationship between concentration and correlation subtraction, making it possible to quantitatively recover Ag nanodot concentrations from high backgrounds in FCS geometries. SAFIRe-FCS should have direct applications to FCS studies in biological systems, making the range of identified modulatable fluorophores ${ }^{9-10,14,21,25,28-30}$ useful labels to observe biological interactions in highly autofluorescent environments or in multiplexed assays.

\section{Supplementary Material}

Refer to Web version on PubMed Central for supplementary material.

\section{Acknowledgments}

The authors gratefully acknowledge support from NIH R21 EB020371 and NIH R01 AI107116, as well as resources from PACE at Georgia Tech.

\section{References}

1. Altman RB, Zheng Q, Zhou Z, Terry DS, Warren JD, Blanchard SC. Enhanced photostability of cyanine fluorophores across the visible spectrum. Nature Meth. 2012; 9:428-429.

2. Zhang X, Xiao Y, Qi J, Qu J, Kim B, Yue X, Belfield KD. Long-Wavelength, Photostable, twophoton excitable BODIPY fluorophores readily modifiable for molecular probes. J Org Chem. 2013; 78:9153-9160. [PubMed: 23984818]

3. Wu X, Zhu W. Stability enhancement of fluorophores for lighting up practical application in bioimaging. Chem Soc Rev. 2015; 44:4179-4184. [PubMed: 25175934]

4. Zheng Q, Juette MF, Jockusch S, Wasserman MR, Zhou Z, Altman RB, Blanchard SC. Ultra-stable organic fluorophores for single-molecule research. Chem Soc Rev. 2014; 43:1044-1056. [PubMed: 24177677]

5. Weissleder R, Ntziachristos V. Shedding light onto live molecular targets. Nature Medicine. 2003; 9:123-128. 
6. Kim SA, Heinze KG, Schwille P. Fluorescence correlation spectroscopy in living cells. Nature Meth. 2007; 4:963-973.

7. Petty JT, Fan C, Story SP, Sengupta B, Sartin M, Hsiang JC, Perry JW, Dickson RM. Optically enhanced, near-IR, silver cluster emission altered by single base changes in the DNA template. Journal of Physical Chemistry B. 2011; 115:7996-8003.

8. Liao Z, Tropiano M, Faulkner S, Vosch T, Sorensen TJ. Time-resolved confocal microscopy using lanthanide centred near-IR emission. RSC Advances. 2015; 5:70282-70286.

9. Richards CI, Hsiang JC, Senapati D, Patel SA, Yu J, Vosch T, Dickson RM. Optically modulated fluorophores for selective fluorescence signal recovery. J Am Chem Soc. 2009; 131:4619-4621. [PubMed: 19284790]

10. Richards CI, Hsiang JC, Dickson RM. Synchronously amplified fluorescence image recovery (SAFIRe). J Phys Chem B. 2010; 114:660-665. [PubMed: 19902923]

11. Richards CI, Choi S, Hsiang JC, Antoku Y, Vosch T, Bongiorno A, Tzeng YL, Dickson RM. Oligonucleotide-stabilized Ag nanocluster fluorophores. J Am Chem Soc. 2008; 130:5038-5039. [PubMed: 18345630]

12. Vosch T, Antoku Y, Hsiang JC, Richards CI, Gonzalez JI, Dickson RM. Strongly emissive individual DNA-encapsulated Ag nanoclusters as single-molecule fluorophores. Proc Natl Acad Sci US A. 2007; 104:12616-12621.

13. Volkov IL, Serdobintsev PY, Kononov AI. DNA-stabilized silver nanoclusters with high yield of dark state. The Journal of Physical Chemistry C. 2013; 117:24079-24083.

14. Hsiang JC, Jablonski AE, Dickson RM. Optically modulated fluorescence bioimaging: Visualizing obscured fluorophores in high background. Accounts of Chemical Research. 2014; 47:1545-1554. [PubMed: 24725021]

15. Elson E, Magde D. Fluorescence correlation spectroscopy. I. Conceptual basis and theory. Biopolymers. 1974; 13:1-28.

16. Schwille P, Haupts U, Maiti S, Webb W. Molecular dynamics in living cells observed by fluorescence correlation spectroscopy with one- and two-photon excitation. Biophys J. 1999; 77:2251-2265. [PubMed: 10512844]

17. Laurence TA, Ly S, Bourguet F, Fischer NO, Coleman MA. Fluorescence correlation spectroscopy at micromolar concentrations without optical nanoconfinement. J Phys Chem B. 2014; 118:96627. [PubMed: 25060197]

18. Schwille, P.; Heinze, KG.; Dittrich, P.; Haustein, E. Two-photon fluorescence correlation spectroscopy. In: Fujimoto, JGF.; Daniel, L., editors. Biomedical Optical Imaging. Oxford University Press; New York, NY: 2009.

19. Elson, Elliot L. Fluorescence correlation spectroscopy: past, present, future. Biophys J. 2011; 101:2855-2870. [PubMed: 22208184]

20. Choi S, Dickson RM, Yu J. Developing luminescent silver nanodots for biological applications. Chem Soc Rev. 2012; 41:1867-91. [PubMed: 22076614]

21. Fan C, Hsiang JC, Dickson RM. Optical modulation and selective recovery of Cy5 fluorescence. ChemPhysChem. 2012; 13:1023-1029. [PubMed: 22086764]

22. Magde D, Elson EL, Webb WW. Fluorescence correlation spectroscopy 2. Experimental realization. Biopolymers. 1974; 13:29-61. [PubMed: 4818131]

23. Krichevsky O, Bonnet G. Fluorescence correlation spectroscopy: the technique and its applications. Reports on Progress in Physics. 2002; 65:251-297.

24. Patel SA, Cozzuol M, Hales JM, Richards CI, Sartin M, Hsiang JC, Vosch T, Perry JW, Dickson RM. Electron transfer-induced blinking in Ag nanodot fluorescence. J Phys Chem C. 2009; 113:20264-20270.

25. Jablonski AE, Vegh RB, Hsiang JC, Bommarius B, Chen YC, Solntsev KM, Bommarius AS, Tolbert LM, Dickson RM. Optically modulatable blue fluorescent proteins. J Amer Chem Soc. 2013; 135:16410-7. [PubMed: 24099419]

26. Petty JT, Zheng J, Hud NV, Dickson RM. DNA-templated Ag nanocluster formation. J Amer Chem Soc. 2004; 126:5207-5212. [PubMed: 15099104]

27. Patel SA, Richards CI, Hsiang JC, Dickson RM. Water-soluble Ag nanoclusters exhibit strong twophoton-induced fluorescence. J Amer Chem Soc. 2008; 130:11602-3. [PubMed: 18686957] 
28. Jablonski A, Hsiang J, Bagchi P, Hull N, Richards CI, Fahrni CJ, Dickson RM. Signal discrimination between fluorescent proteins in live cells by long-wavelength optical modulation. $\mathrm{J}$ Phys Chem Lett. 2012; 3:3585-3591. [PubMed: 23419973]

29. Richards CI, Hsiang JC, Khalil AM, Hull NP, Dickson RM. FRET-enabled optical modulation for high sensitivity fluorescence imaging. J Amer Chem Soc. 2010; 132:6318-6323. [PubMed: 20397664]

30. Sarkar S, Fan C, Hsiang JC, Dickson RM. Modulated fluorophore signal recovery buried within tissue mimicking phantoms. J Phys Chem A. 2013; 117:9501-9509. [PubMed: 23692258] 


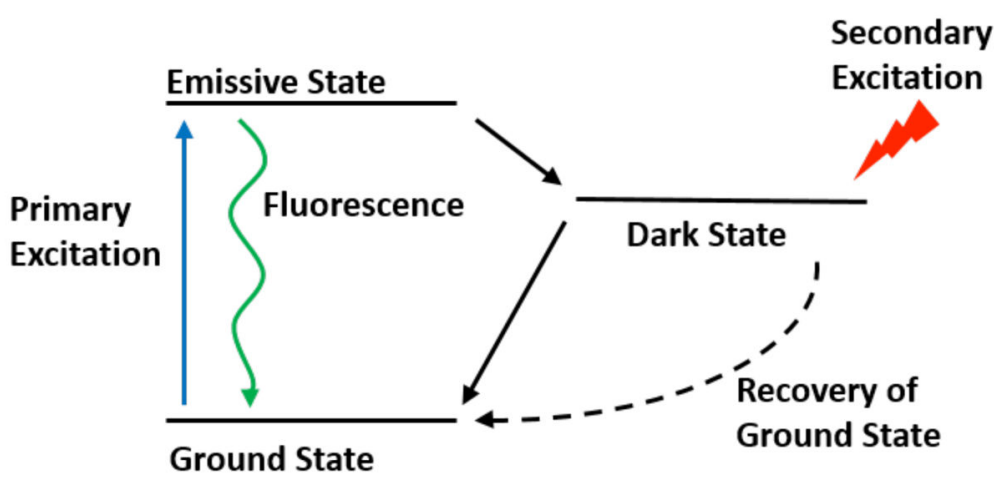

Figure 1.

Jablonski diagram of ground, emissive, and dark states for 630-nm Ag cluster emitters. Coexcitation at $805 \mathrm{~nm}$ (red arrow) depopulates the dark state faster than its natural decay rate to more rapidly recover ground state population and increase overall 630nm fluorescence. 


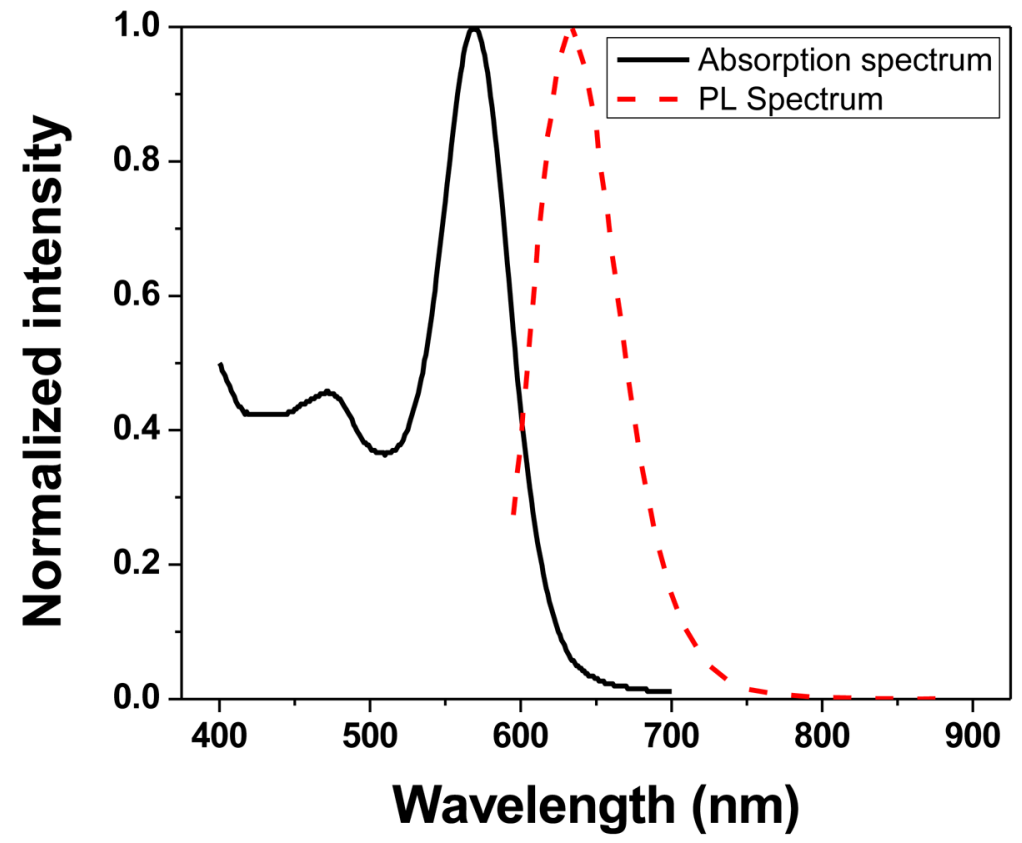

Figure 2.

Absorption and emission spectra for $630 \mathrm{~nm} \mathrm{Ag}$ cluster emitter, synthesized with $5^{\prime}$ CCCCAACTCC-3' DNA. 


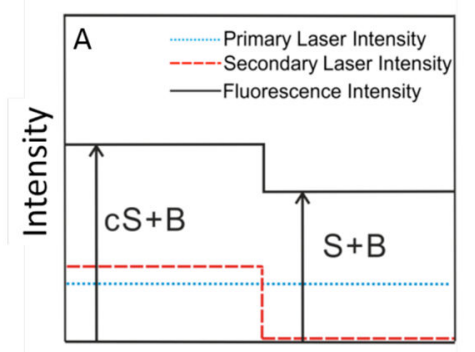

Time

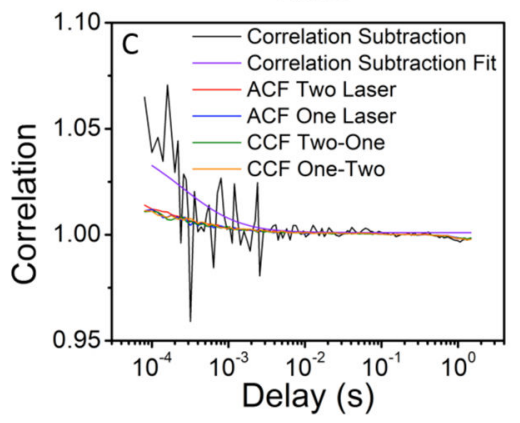

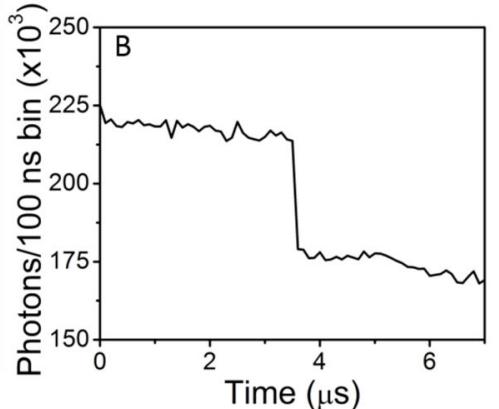

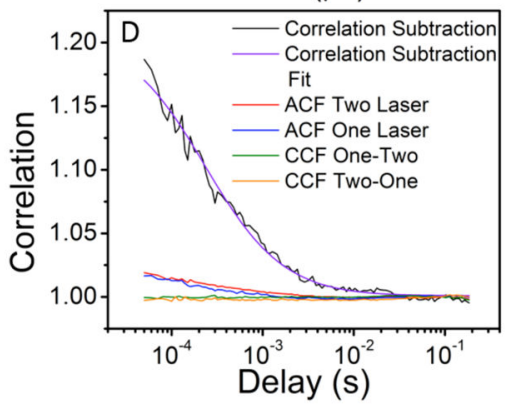

Figure 3.

Correlation subtraction of modulated $630 \mathrm{~nm}$ emitters in a 1:1 concentration of Texas Red background, illuminated with $594 \mathrm{~nm}$ primary and $805 \mathrm{~nm}$ secondary laser intensities of 400 $\mathrm{W} / \mathrm{cm}^{2}$ and $70 \mathrm{~kW} / \mathrm{cm}^{2}$, respectively. A) Schematic of a single modulation period (microtime). The primary cw excitation source is maintained at constant intensity while the secondary source is intensity modulated with a square wave, leading to a modulated fluorescence signal. B) Experimental microtime window of $100 \mathrm{kHz}$ modulation cycle in counts. C) $100 \mathrm{kHz}$ modulated fluorescence data from $\mathrm{B}$ ) is divided into photon trajectories corresponding to single $(\mathrm{S}[\mathrm{t}]+\mathrm{B}[\mathrm{t}])$ and dual $(\mathrm{cS}[\mathrm{t}]+\mathrm{B}[\mathrm{t}])$ excitation, and correlations are performed according to $\mathrm{Eq} 2$. While all correlations are individually normalized for presentation on the same graph, only the final output from Eq. 2 is normalized and fit to Eq. 1. Recovered diffusion constants from correlation subtracted data in $\mathrm{C}$ and $\mathrm{D}$ are $(2.0 \pm 0.7)$ $\times 10^{-10} \mathrm{~m}^{2} \mathrm{~s}^{-1}$ and $(1.8 \pm 0.5) \times 10^{-10} \mathrm{~m}^{2} \mathrm{~s}^{-1}$, respectively. D) Correlations from $1 \mathrm{~Hz}$ modulation of the same sample as in C. Zoomed-in plots of the correlation functions in $\mathrm{C}$ and D are presented in Figures S3 B and A, respectively. 

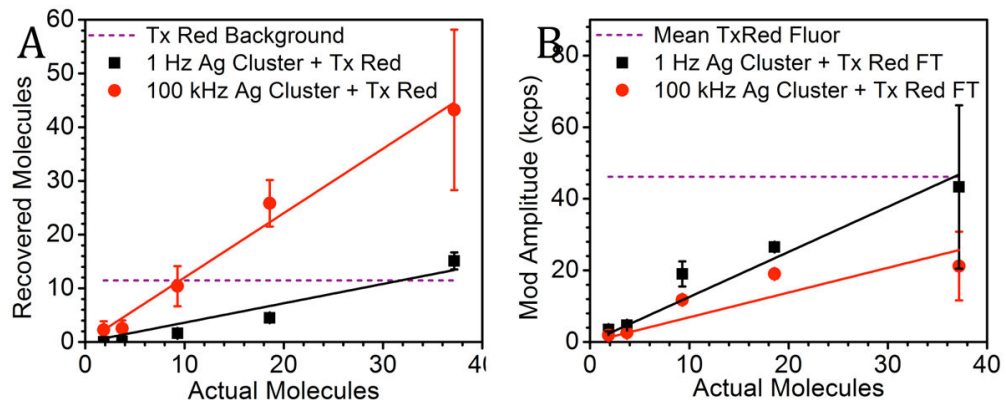

Figure 4.

Concentration recovery of $630 \mathrm{~nm}$ emitters with Texas Red background, illuminated with $594 \mathrm{~nm}$ primary and $805 \mathrm{~nm}$ secondary laser intensities of $400 \mathrm{~W} / \mathrm{cm}^{2}$ and $70 \mathrm{~kW} / \mathrm{cm}^{2}$, respectively. A) Plot of actual concentration vs correlation subtraction concentration at 100 $\mathrm{kHz}$ and $1 \mathrm{~Hz}$ modulation frequencies. B) Plot of the Fourier transform amplitude vs number of molecules in the FCS excitation volume for $1 \mathrm{~Hz}$ and $100 \mathrm{kHz}$ modulation frequencies. Error bars result from the standard deviation resulting from triplicate runs. 


\section{Table 1}

Photophysical properties of $630 \mathrm{Ag}$-DNA clusters

\begin{tabular}{|c|c|c|c|}
\hline Emission & $\Phi_{\mathbf{F}}$ & $\boldsymbol{\varepsilon}, \mathbf{1 0}^{\mathbf{5}} \mathbf{M}^{\mathbf{- 1}} \mathbf{c m}^{\mathbf{1}}$ & $\boldsymbol{\tau}_{\text {off }}, \boldsymbol{\mu s}$ \\
\hline $630 \mathrm{~nm}$ & $0.18 \pm 0.03$ & $2.6 \pm 0.1$ & $11 \pm 1$ \\
\hline
\end{tabular}

